\title{
ANOMALOUS RESISTIVITY RESULTING FROM ELECTROSTATIC ION CYCLOTRON TURBULENCE
}

\author{
J.A. IONSON* \\ Space Physics Research Laboratory, Department of Atmospheric and Oceanic Science \\ University of Michigan, Ann Arbor, MI 48109, USA
}

Received 12 April 1976

\begin{abstract}
An expression is found for the collisionless electrical resistivity resulting from a current driven electrostatic ion cyclotron instability.
\end{abstract}

Over the past few years a large amount of work has been devoted to the study of transport phenomena in a linearly unstable, nonlinearly stable plasma. In particular, collective resistivity resulting from a current driven instability has dominated the scene. However, investigations have leaned quite heavily toward the ion-acoustic instability $[1,2,3]$ and Buneman instability [4] which develop only when critical criteria have been satisfied. Magnetoplasmas which are found in natural systems often do not satisfy these criteria and it is important to investigate other possible sources of plasma turbulence.

In its simplest form, collective (anomalous) resistance can be attributed to teh scattering or stochastic deceleration of an electron current due to collective field fluctuations. For a linearly stable plasma, electrical resistance is determined from random thermal fluctuations; and are represented by the plasma parameter $g \equiv\left(n_{\mathrm{e}} \lambda_{\mathrm{De}}^{3}\right)^{-1} \sim \epsilon_{\mathrm{th}}$ where $\epsilon_{\mathrm{th}}$ is the energy density of field fluctuations normalized to the plasma's macroscopic kinetic pressure $n_{\mathrm{e}} T_{\mathrm{e}}$. Classical resistivity can therefore be represented by $\eta_{\mathrm{c}} \sim 4 \pi \mathrm{g} / \omega_{\mathrm{pe}} \sim 4 \pi \epsilon_{\mathrm{th}} / \omega_{\mathrm{pe}}$ where $\epsilon_{\mathrm{th}} \ll 1$. When a plasma becomes unstable to collective fluctuations, the normalized energy density of these waves eventually saturates by some nonlinear mechanism to a level $\epsilon_{\mathrm{s}}$. Popular mechanisms developed for electrostatic turbulence in a magnetoplasma are resonance broadening due to both configuration and velocity space diffusion $[5,6,7]$ and induced nonlinear scattering [8]. Saturation usually takes place in a time $t_{0} \sim \bar{\omega}^{-1}$ where $\bar{\omega}$ is a frequency characteristic of the turbulence. Provided this turbulence is resonant with the bulk of the electron current, electrons will be scattered primarily from collective fluctuations rather than thermal fluctuations. After a time $t_{1} \sim\left(\epsilon_{\mathrm{s}} \bar{\omega}\right)^{-1}$, however, relaxation of the ambient distribution must be accounted for unless there is some mechanism with retards plateau formation.

We are concerned primarily with turbulence generation in an isothermal, isotropic plasma where the drift velocity is below that of the ion-acoustic threshold. As found by Drummond and Rosenbluth [9] electrostatic ion cyclotron turbulence (E.I.C.T.) is a likely candidate. General properties of E.I.C.T. are as follows:
(a) $k^{2} \rho_{\mathrm{ci}}^{2} \sim 0(1)$;
(b) $k^{2} \rho_{\mathrm{ce}}^{2} \ll 1$
(c) $k \sim k_{\perp} \gg k_{\|}$,

where $\rho_{\mathrm{c} \alpha}^{2} \equiv v_{\alpha}^{2} / \Omega_{\alpha}^{2}, v_{\alpha}^{2} \equiv 2 T_{\alpha} / m_{\alpha}$ and $\Omega_{\alpha} \equiv q_{\alpha} B_{0} / m_{\alpha} c$. For an isothermal, isotropic plasma the wave of maximum growth is such that $\bar{k}_{1} / \bar{k}_{\|} \sim 10, \omega-\Omega_{\mathrm{i}} \sim 0.25 \Omega_{\mathrm{i}}$ with a critical drift $v_{\mathrm{c}} \sim 13 v_{\mathrm{i}}$ [10].

The general velocity space diffusion tensor for electrostatic waves in a magnetoplasma is given by

$\left(D_{\perp \perp}^{\alpha}, D_{\perp \|}^{\alpha}, D_{\|\|}^{\alpha}\right)=\frac{e_{\alpha}^{2}}{m_{\alpha}^{2}} \sum_{n} \int \mathrm{d}^{3} K \frac{\left|E_{K}^{2}\right| J_{n}^{2}\left(\lambda_{\alpha}\right)}{\mathrm{i}\left(-\omega+k_{\|} v_{\|}+n \Omega_{\alpha}\right)}\left(\frac{k_{\perp}^{2} n^{2}}{k^{2} \lambda_{\alpha}^{2}}, \frac{k_{\|} k_{\perp} n}{k^{2} \lambda_{\alpha}}, \frac{k_{\|}^{2}}{k^{2}}\right)$,

$D_{\perp \|}^{\alpha}=D_{\| \perp}^{\alpha}, \lambda_{\alpha} \equiv k_{\perp} v_{\perp} /\left|\Omega_{\alpha}\right|, J_{n} \equiv$ Bessel function of order $n$ where $1 /\left(\omega-k_{\|} v_{\|}-n \Omega_{\alpha}\right) \rightarrow P\left(\omega-k_{\|} v_{\|}-n \Omega_{\alpha}\right)-\mathrm{i} R_{\alpha}$ with a resonance function defined by

* Presently at the Department of Physics and Astronomy, The University of Maryland, College Park, Maryland 20742, USA. 
$R_{\alpha} \equiv \operatorname{Re} \int_{0}^{\infty} \mathrm{d} \tau \exp \left[\mathrm{i}\left(\omega-k_{\|} v_{\|}-n \Omega_{\alpha}\right) \tau-\frac{1}{2}\left\langle\left(k \cdot \delta X_{1}^{\alpha}\right)^{2}\right\rangle\right]$

$\left\langle\left(k \cdot \delta X_{1}^{\alpha}\right)^{2}\right\rangle$ represents the average mean square deviation from the mean of the particle (species $\alpha$ ) trajectories in the turbulent fields given by

$\left\langle\left(k \cdot \delta X_{1}^{\alpha}\right)^{2}\right\rangle \equiv 2\left(k \cdot \stackrel{\leftrightarrow}{D_{\alpha}^{*}} \cdot k\right) \tau$;

$\overleftrightarrow{D_{\alpha}^{*}}$ appearing in Weinstock's [6], eq. (33). Note that a small, real non-linear frequency shift has been neglected in $R_{\alpha}$ [11]. In this note we assume that the magnetic field is sufficiently large to satisfy $\Omega_{\alpha} \tau_{\mathrm{c}} \gg 1$ where $\tau_{\mathrm{c}}$ is the correlation time of the electrostatic field fluctuations given by

$\tau_{\mathrm{c}} \equiv \int_{0}^{\infty} \mathrm{d} s \frac{\langle E(x, t) \cdot E(x(t-s), t-s)\rangle}{\left\langle|E(x, t)|^{2}\right\rangle}$

which assumes a stationary spectrum. Once the condition $\Omega_{\alpha} \tau_{c} \gg 1$ is satisfied, Weinstock's modified diffusion coefficient $\overleftrightarrow{D}_{\alpha}^{*}$ is reduced to a form such that

$\left(k \cdot \overleftrightarrow{D}_{\alpha}^{*} \cdot k\right) \tau=\frac{1}{3} k_{\|}^{2} D_{\|\|}^{\alpha} \tau^{3}+\frac{k_{\perp} k_{\|}}{\Omega_{\alpha}} D_{\| \perp}^{\alpha} \tau^{2}+\frac{k_{\perp}^{2}}{\Omega_{\alpha}^{2}} D_{\perp \perp}^{\alpha} \tau$.

The saturated spectrum is then found to be that which results from an anomalous nonlinear damping of the waves, related to stochastic ion orbit diffusion in configuration space. This particular damping mechanism dominates longitudinal trapping (related to stochastic orbit diffusion in velocity space) whenever

$=\frac{k_{\perp}^{2} D_{\perp \perp}^{i}}{\Omega_{\mathrm{i}}^{2}} \gg\left(k_{\|\|}^{2} D_{\|\|}^{i}\right)^{1 / 3}$ or $\epsilon_{\mathrm{s}} \gg\left(k_{\|} / k_{\perp}\right)^{4}\left(\Omega_{\mathrm{i}} / \omega_{\mathrm{pi}}\right)^{2}$.

Therefore, if the ratio $\Omega_{\mathrm{i}} / \omega_{\mathrm{pi}}$ becomes large enough, longitudinal trapping could very well result in a lower saturated energy. With such a mechanism, formation of a field aligned ion tail would be the dominant stabilization mechanism. We will assume, however, that $\Omega_{\mathrm{i}} / \omega_{\mathrm{pi}}$ is small; giving rise to negligible longitudinal trapping. For electron drifts which satisfy

$\frac{V_{\mathrm{d}}-\bar{\omega} / \bar{k}_{\|}}{V_{\mathrm{e}}} \ll 1 \quad$ or $\quad V_{\mathrm{d}} \ll 1.3 V_{\mathrm{e}}$

Dum and Dupree [7] have found that the saturated energy density is independent of the drift. Since it is this region in which we are interested, their results will be used. For an isothermal plasma, the energy density saturates at a level independent of the drift and is found to be

$\epsilon_{\mathrm{s}} \sim 0.1\left(\Omega_{\mathrm{i}} / \omega_{\mathrm{pi}}\right)^{2} \quad$ for $\quad T_{\mathrm{e}} \sim T_{\mathrm{i}}$.

Using this saturated level of turbulence, we can now develop an expression for electrical resistivity.

Taking the first moment of

$-\frac{e}{m_{\mathrm{e}}} E_{\|} \frac{\partial f_{\mathrm{e}}}{\partial v_{\|}}-\frac{e}{m_{\mathrm{e}}}\left(\frac{v_{\perp} B_{0}}{c}\right) \frac{\partial f_{\mathrm{e}}}{\partial v_{\perp}}=\frac{\partial}{\partial v_{\|}} D_{\|}^{\mathrm{e}}\left(\mathrm{v}, \epsilon_{\mathrm{s}}\right) \frac{\partial f_{\mathrm{e}}}{\partial v_{\|}}$,

gives

$E_{\|}=-\frac{m_{\mathrm{e}}}{n_{\mathrm{e}} e} \int \mathrm{d}^{3} v D_{\|}^{\mathrm{e}}\left(v, \epsilon_{\mathrm{s}}\right) \frac{\partial f_{\mathrm{e}}}{\partial v_{\|}}$,

where the time scale is such that the system is in a steady state with a saturated level of turbulence. Although longitudinal trapping of the electrons does not affect the saturated energy level, these effects must be incorporated when 
investigating the stochastic deceleration of an electron beam. This can be confirmed by noting that an estimate for the half width of the velocity resonance gives

$w_{1 / 2}^{\mathrm{e}} \sim \frac{1}{k_{\|}}\left(k_{\|}^{2} D_{\|}^{\mathrm{e}}\left(v_{\mathrm{c}}, \epsilon_{\mathrm{s}}\right) / 3\right)^{1 / 3} \sim 0.4 v_{\mathrm{e}}$,

where $v_{\mathrm{c}}$ is a critical velocity at which the electron diffusion coefficient begins to peak $\left(\bar{\omega}-\bar{k}_{\|} v_{\mathrm{c}}\right) \sim$

$\left(\bar{k}_{\|}^{2} D_{\|}^{\mathrm{e}}\left(v_{\mathrm{c}}, \epsilon_{\mathrm{s}}\right) / 3\right)^{1 / 3}$. Clearly, the bulk of the electrons are stochastically decelerated. Approximating the electron resonance function by a gaussian

$R_{\mathrm{e}}=1 /\left(2 \pi k_{\|}^{2} w_{1 / 2}^{\mathrm{e}^{2}}\right)^{1 / 2} \exp \left[-\left(k_{\|} v_{\|}-\omega\right)^{2} / 2 k_{\|}^{2} w_{1 / 2}^{\mathrm{e}^{2}}\right]$,

the integration in (11) can be performed to give an anomalous resistivity

$\eta_{\mathrm{a}}=0.06\left(\Omega_{\mathrm{i}} / \omega_{\mathrm{pj}}\right)\left(1-12 v_{\mathrm{i}} / v_{\mathrm{d}}\right) \omega_{\mathrm{pe}}^{-1}$.

Implicit within this expression are the criteria given in eq. (1), (8), (9) and (12).

Eq. (14) holds in an isothermal, maxwellian, magnetoplasma provided the electron drift exceeds $13 v_{\mathrm{i}}$.

The author wishes to thank Professor R.S. Ong and Dr. E.G. Fontheim for many helpful discussions. This research has been supported by the National Science Foundation under Grant No. ATM 73-00322 A01 and by the National Aeronautics and Space Administration under Grant No. NGR 23-005-015.

\section{References}

[1] D. Biskamp and K. Chodura, 4th Int. Conf. on Plasma physics and controlled nuclear fusion research, Madison, 1971. Paper CN-28/E-17.

[2] S.P. Gary and J.W.M. Paul, Phys. Rev. Lett. 26 (1971) 1097.

[3] M.Z. Caponi and R.C. Davidson, Phys. Rev. Lett. 31 (1973) 86.

[4] S. Ichimaru, J. Phys. Soc. Japan 39 (1975) 261.

[5] T.H. Dupree, Phys. Fluids 11 (1968) 2680.

[6] J. Weinstock, Phys. Fluids 13 (1970) 2308.

[7] C.T. Dum and T.H. Dupree, Phys. Fluids 13 (1970) 2064.

[8] R.C. Davidson, Methods in nonlinear plasma theory (Academic Press, New York and London, 1972).

[9] W.E. Drummond and N.M. Rosenbluth, Phys. Fluids 5 (1962) 1507.

[10] J.M. Kindel and C.F. Kennel, J. Geophys. Res. 76 (1971) 3055.

[11] J. Weinstock, Phys. Fluids 15 (1972) 454. 